Research Notes

\title{
Analyses of the Interleukin32 Transcript Variants in Breast Cancer Cell Lines
}

\author{
Kayla Burrell and Audrey Player \\ Department of Biological Sciences, Texas Southern University, Houston Texas, USA
}

\author{
Article history \\ Received: 14-09-2016 \\ Revised: $13-10-2016$ \\ Accepted: 15-10-2016 \\ Corresponding Author: \\ Audrey Player \\ Department of Biological \\ Sciences, Texas Southern \\ University, Houston Texas, \\ USA \\ Tel: 713.313 .7837 \\ Fax: 713.313.7932 \\ Email: playeran@tsu.edu
}

\begin{abstract}
Recent data show the IL32 gene has at least nine transcript variants. The aim of this current study is to characterize the different transcript variants based on the latest sequencing data deposited in the National Center for Biotechnology Information (NCBI) and determine which variants are responsible for the differential pattern of gene expression previously observed in MCF7 compared to MDA MB231 cell lines. Analyses of the nine transcript variants showed their sequences were incredibly similar. Other than variant 9 , all of the variants differed from variant 1 by deletions. PCR analyses showed that the longer transcript variants contributed more to differential gene expression observed in the MDA MB231 compared to MCF7 cell lines. Because of the similarities between the variant sequences, when determining differential expression in the breast cell lines, investigators should consider strategies that target analyses of a combination of the longer IL32 transcript variants.
\end{abstract}

Keywords: IL32, Triple Negative Breast Cancer, Transcript Variants

\section{Introduction}

The Interleukin32 (IL32) gene was first identified by (Dahl et al., 1992) in natural killer and T cells. The gene codes for a cytokine and is located on chromosome 16p13.3. Even though IL32 is a cytokine, its sequence is not homologues to the other known cytokines. Analyses of the corresponding protein show it contains a tyrosine sulfation site, 3 potential N-myristoylation sites, putative phosphorylation sites and RGD binding sequences (Felaco et al., 2009). Related to its function as a cytokine, studies demonstrate a role of IL32 in pro-inflammatory processes (Kobayashi et al., 2009; Joosten et al., 2013). Data also show the IL32 gene stimulates TNFa, IL10 and IL8 (Kang et al., 2009; Kim et al., 2002; 2005). Like other cytokines IL32 is expressed in immune type cells, but the gene is also expressed in endothelial and epithelial cells related to mechanisms related to differentiation and cancers (Mabilleau and Sabokbar, 2009; Yue et al., 2010; Ishigami et al., 2013; Trojan et al., 2005). Supporting a possible role in cancers, (Kim et al., 2002) described IL32 gene expression in A549 lung cells following over-expression of IL18 receptor $\beta$ gene. In addition, Oh et al. (2005) found that IL32 was overexpressed in gastric cancer while another study (Park et al., 2013) identified IL32 overexpression in breast cancer cell lines. The authors showed that IL32 $\beta$ stimulates migration of MDA MB231 and MCF7 via vascular-endothelial-growth-factor-signal-transducer-andactivator (VEGF-STAT3), suggesting both cell lines were positive for the related receptors and most importantly, an increased level of IL32 in both cell lines was correlated with tumor progression. The studies also found that IL32 expression was consistently correlated with the expression of epithelial-to-mesenchymal (EMT) markers, slug and vimentin confirming a role of IL32 gene in signaling processes related to both differentiation and migration. This was the first observation of IL32 gene expression in the triple negative breast cancer (TNBC; MDA MB231) and luminal breast cancer (MCF7) cell lines. In further support of the role of IL32 in tumors, using a xenograft model system, Wang et al. (2015) showed that IL32 gene increased the rate of proliferation and inhibited apoptosis in breast cancer cells and Nold-Petry et al. (2015) showed that IL32 promotes angiogenesis in breast cancer cells. Collectively these data suggest a role of IL32 in differentiation and tumor pathogenesis supporting its study in events related to cancers.

Park et al. (2013) found that over expression of IL32 $\beta$ correlated with tumor progression in breast cells, however, compared to other tissues, less is known about IL32 in breast samples. Although their study show that luminal and TNBC cell types express IL32 gene, studies in our laboratory demonstrate substantial differential gene expression of IL32 in basal-like/TNBCs compared 
to normal and luminal breast cancer cell lines and patient samples (Player et al., 2014). Since our initial observations, we have performed studies to better characterize the IL32 gene in breast cancers.

Data show that as of June 2016, there are nine IL32 transcript variants, so not only is there an attempt to better characterize IL32, but a primary goal of our studies is to determine which of the variants are responsible for the differential pattern of expression observed in TNBC samples. In the event IL32 proves to be clinically relevant in breast cancer or inflammatory related diseases, it will ultimately be important to recognize the differences between the different isoforms; and specifically related to breast cancers these experiments will identify which of the isoforms to target in efforts to better understand the function of the gene in breast. Outlined in this current study, we performed analyses of the IL32 transcript variants and found that their sequences were incredibly similar. Based on nucleotide regions found to be unique per isoforms, we then generated PCR primers and determined differential gene expression of the variants in luminal compared to a TNBC cell line. Because of their incredible sequence similarity, in most cases we were not able to identify unique regions corresponding to the individual variants, making it difficult to interpret gene expression levels based on each isoform. Our data show that many of the variants are indeed transcribed in the breast cells, so it might not be possible to completely separate their activity and subsequently target one isoform to modulate the gene's transcript expression. Our data also show that combinations of the transcript variants are expressed in our breast cancer cell lines and variants with longer sequences contribute more to the differential pattern of expression compared to variants with deletions. Data presented in this study represent the most detailed analyses of the nine IL32 variants since the corrected sequences were deposited in NCBI. Transcript variant 9 has only recently been characterized, as a result no other study contains information related to comparison of this variant to all other transcript variants. Based on analyses of the sequences currently deposited in the National Center for Biotechnology Information (NCBI), we find that the nine variants code for five closely related forms of the IL32 protein. Previous data suggest the IL32 is involved in the pathogenesis of inflammatory diseases and breast and other cancers, as a result data presented in this current study are important for better understanding the IL32 gene.

\section{Materials and Methods}

\section{Cell Lines Selection}

The MCF7 cell was chosen to represent the luminal cell line and the MDA MB231 was chosen to represent TNBC cell line. The cell lines were purchased from
ATCC (Manassas, VA). The cells were cultured in Dulbecco's Modified Eagle Minimum essential media (DMEM) supplemented with penicillin and $10 \%$ serum in a $37^{\circ} \mathrm{C}$ degree incubator with $5 \% \mathrm{CO} 2$ as suggested by the supplier.

\section{RNA Extraction, Analysis and Generation of $c D N A$}

The cells were grown to approximately $90 \%$ confluence and harvested for extraction of total RNA. RNA was extracted using the TrizolTM (Thermo-Fisher, Grand Island, NY) method. The RNA quality was examined following spectrophotometer analysis (via assessment of the A260/280 ratio) and by visual analyses following separation by electrophoresis on a $1 \%$ agarose-formaldehyde gel. One microgram of total RNA was converted to copy DNA (cDNA) using the iScript cDNA kit (Bio-Rad, Hercules $\mathrm{CA})$ as suggested by the manufacturer. The cDNA samples were diluted five-fold with water and stored at $20^{\circ} \mathrm{C}$ until used in experimental analyses.

\section{Analyses of IL32 Gene, Primer Design, PCR and Gel Electrophoresis}

The IL32 transcript variants and protein sequences were analyzed using NCBI tools including the Basic Local Alignment Search Tool (BLAST; https://blast.ncbi.nlm.nih.gov/Blast.cgi), University of California Santa Cruz Genome Brower (UCSC Genome Browser; https://genome.ucsc.edu/) and the Multalin program (Corpet, 1988). The nucleotide and protein sequences corresponding to IL32 were retrieved from NCBI (GRCh38.p7 build). Nucleotide and protein sequences corresponding to the nine IL32 isoforms were aligned and compared using the Multalin program and BLAST to characterize the various forms and identify unique regions related to each. The unique nucleotide regions were identified and PCR primers were generated using the Primer3 program (Rozen and Skaletsky, 2000). BLAST and the In Silico PCR program of the UCSC Genome Browser were used to validate the specificity of the PCR primer sequences chosen. These programs were also used to analyze the amplicons resulting from forward and reverse primer hybridizations against the entire genome to determine their position along the chromosomes. Primer sequences were synthesized by IDTDNA (Coralville IA).

For the PCR reactions, approximately 10 nanograms (ng) of cDNA (MCF7 or MDA MB231 respectively) was mixed with $10 \mathrm{uL}$ of Amplitaq GoldTM (Life Technologies, Waltham MA), 10uM each of the forward and reverse gene primers and water up to $20 \mathrm{uL}$. PCR conditions were carried out at $95^{\circ} \mathrm{C} 5 \mathrm{~min}$ for one cycle, then 30 cycles at $95^{\circ} \mathrm{C}$ for $30 \mathrm{sec}, 58^{\circ} \mathrm{C}$ for $90 \mathrm{sec}$ and $70^{\circ} \mathrm{C}$ for $90 \mathrm{sec}$, followed by a final extension of $70^{\circ} \mathrm{C}$ for $5 \mathrm{~min}$. PCR products were separated by electrophoresis on a $1.0 \%$ agarose gel. 


\section{Assessment of the Performance of the Different Primer Pairs}

The transcript variants responsible for differential expression observed in MDA MB231 compared to MCF7 were determined by comparing the transcript levels generated by PCR analyses following use of the specific primer sets. The overall intensity of the amplicons generated in MDA MB231 was compared to the intensity of the amplicons generated in MCF7, each adjusted for the control gene transcript levels. We compared amplicon amounts generated for (a) primers1/MRPL19 for MDA MB231 -primer1/MRPL19 for MCF7, (b) primers2/MRPL19 for MDA MB231 primer2/MRPL19 for MCF7 (c) primers3/MRPL19 for MDA MB231 -primer3/MRPL19 for MCF7, (d) primers4/MRPL19 for MDA MB231 -primer4/ MRPL19 for MCF7 and (e) primers5/MRPL19 for MDA MB231 - primer5/ MRPL19 for MCF7. The amount of amplicon generated for each primer set was determined by densitometer analyses using the Bio-Rad ChemDoc data analysis software. Primer sets were ranked based on the differential expression of IL32 transcripts in MDA MB231 compared to MCF7 samples.

\section{Results}

\section{Comparisons between the nine IL32 Transcript Variant Sequences}

We previously showed that IL32 was differentially expressed in basal-like/TNBC compared to normal and luminal breast cancer cell lines and clinical samples (Player et al., 2014). For the current study, our goal was to better characterize the transcript variants responsible for the differential pattern of gene expression observed in those samples. As of June 2016 nine IL32 transcript variants have been identified ranging in size from 880 to 1,243 base pair mRNA (corresponding to transcript variants 7 and 9, respectively). The list of the transcript variants and the size of each isoform is given in Table 1. The Greek symbol designations were retrieved from current NCBI entries listed for each variant. The relative intron: Exon distributions of the nine transcript variants are presented in Fig. 1. The exons of each variant is compared to exon sequences in transcript variant 1 . Transcript variant 1 contains eight exons and is considered the canonical sequence. All other variants contain five to seven exons. When aligned, analysis of these data show incredible sequence similarity between all of the transcript variants, with the least similarity between exon two of transcript variant 1 , compared to exon one of variants $3,6,5$ and 9 . Overall, nucleotide sequence analyses show closer similarity between transcript variants 1, 2, 3, 4 and 8 and 5 and 6 and less between 7 and 9 (Fig. 1 and Table 2). Transcript variants 4 and 7 are among the shortest variants with variant 4 lacking exon two and a substantial portion of exon eight and variant 7 lacking exons one, two and six compared to transcript variant 1 . Variants 5 and 6 are also short isoforms, lacking exons one and five compared to variant 1. Except for the aforementioned differences observed in exon two of variant 1, the differences between the variants are mainly in the form of deletions; transcript variant 9 however shows additional nucleotide sequences in exons four, five and eight.

\section{Comparisons between the Corresponding Protein Sequences}

Analysis of the protein sequences show that there are five different IL32 protein sequences corresponding to the nine transcript variants (Fig. 2). The Multalin program is used to compare the sequences but it can also be used to position sequences based on relative similarity. Based on the protein sequences and their positions following Multalin, transcript variants 1, 2, 3 and 8 all represent one protein; variants 5 and 6 represent a different protein. Transcript variants 4, 7 and 9 each represent a different protein. Based on size, the protein coded from transcript variant 9 is the longest at 234 amino acids, followed by the protein coded from variants $1,2,3$ and 8 . Next in size is the protein coded from variant 7 , then the protein coded from variants 5 and 6 . The protein coded from variant 4 is the smallest at 131 amino acids. This information is summarized in Table 2.

$P C R$ and Gel Electrophoresis Analysis of the Transcript Variants in MDA MB231 Compared to MCF7

Our initial goal was to compare the sequences associated with the nine transcript variants, identify unique regions that would allow for PCR analyses of the individual variants and use the primer sets generated from the analyses for comparison of transcript levels in MDA MB231 compared to the MCF7 cell line. These data would allow us to identify which variants are expressed in the two cell lines, hence responsible for the differential pattern of gene expression observed. However we found that there was incredible sequence similarity between the IL32 transcript variants that would not allow for analyses of the individual variants. Based on their nucleotide sequences we were able to design five sets of PCR primers corresponding to the different combinations of isoforms. Supplemental Table 1 lists the primer sets, the transcripts recognized by the particular primers and the size of the amplicons generated by each. In Silico PCR was performed to validate the location on the chromosome and the amplicon sizes. Primer set 1 was used in our initial discovery experiments. Using this primer set we were able to identify differences in transcript levels in basallike/TNBC compared to normal and luminal breast cancer cell lines and clinical samples. 


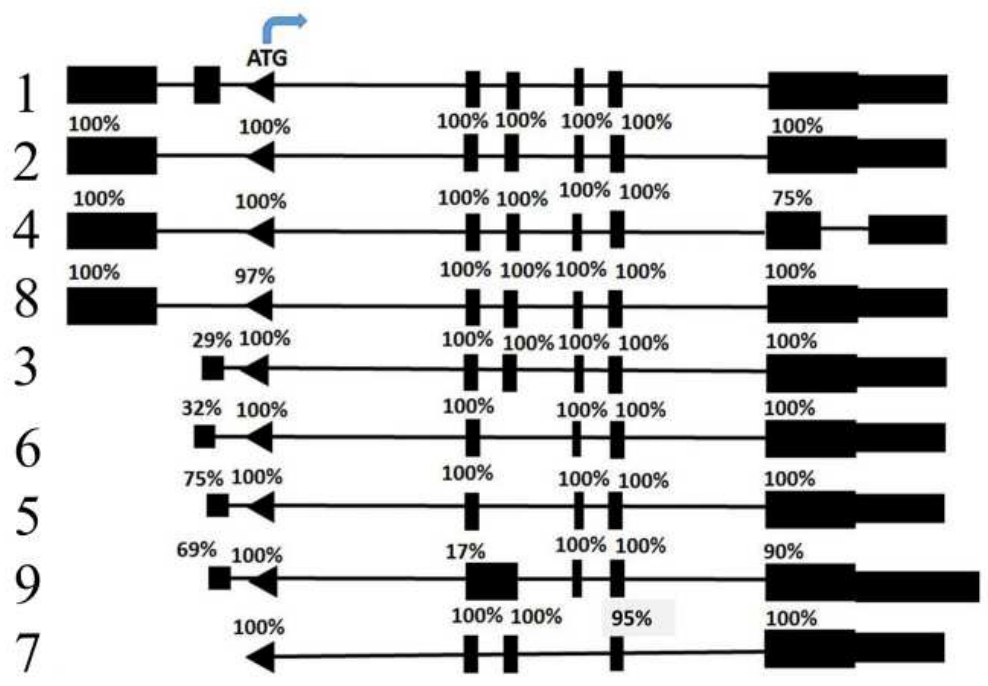

Fig. 1. Intron: Exon display of the nine IL32 transcript variants. The nucleotide data (and the original figure) was retrieved from NCBI (June 2016). The percent similarity between the different exons was determined using Multalin. The canonical sequence is transcript variant 1 with eight exons. The ATG sequence designating the protein start site on exon three of variant 1

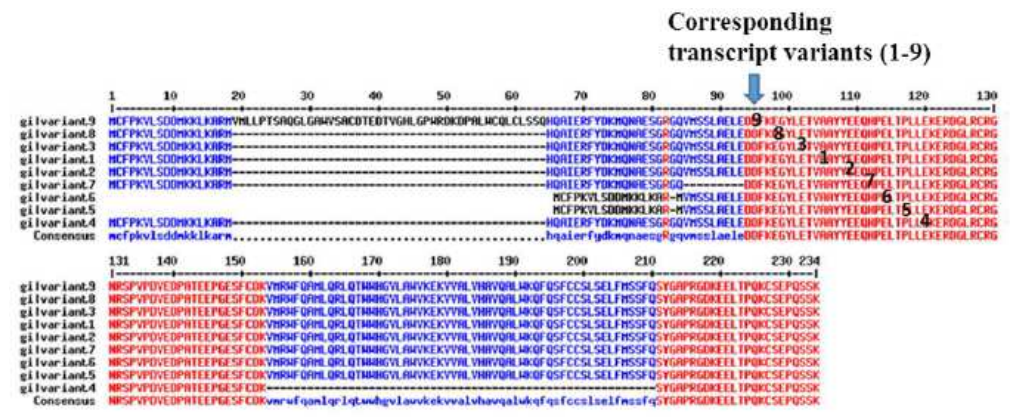

Fig. 2. Comparison of the protein sequences corresponding to the nine different transcript variants. The comparisons between the proteins were generated by Multalin program. Red corresponds to high consensus, blue corresponds to lower consensus and black corresponds to neutral representing less consensus. The variants corresponding to the particular proteins are also listed

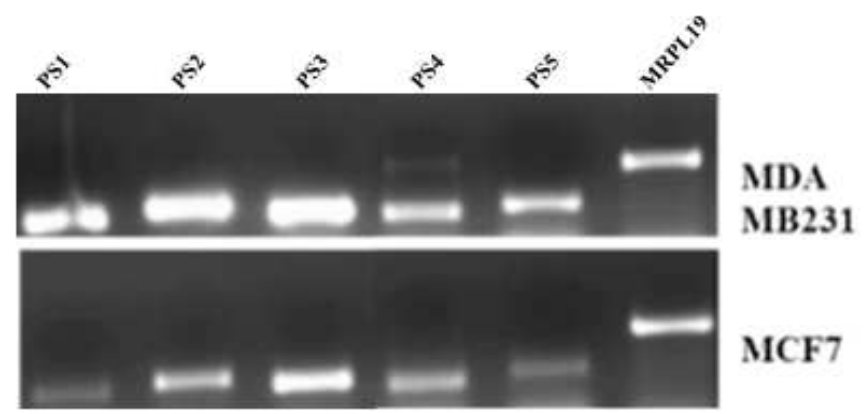

Fig. 3. Analysis of PCR results of different transcript variant sets in MDA MB231 compared to MCF7. MRPL19 represents control gene for both cell line preparations. Primer set (PS)

PCR analysis of the different primer sets in MDA MB231 compared to MCF7 showed that each demonstrated some level of differential expression in the cell lines. However, the transcript variants demonstrated varying levels of differential expression (Fig. 3). The densitometer analyses show that the greatest difference in gene expression is found with primer set one (corresponding to variants $1,2,3,5,6,8$ and 9) and primer set five (corresponding to variant 9 only). These data suggest that variants $1,2,3$ and 8 and variants 5 and 6 and variant 9 are more active in MDA MB231 cells leading to differential expression compared to MCF7. 
Table 1. Descriptions of the nine Il32 transcript variants. Data were obtained from NCBI

Analysis of IL32 transcript variants

\begin{tabular}{lll} 
& & Length (base pairs) \\
Reference (NCBI) & Description & 1157 \\
\hline NM_001012631.1 & transcript variant 1 & 1090 \\
NM_004221.4 & transcript variant 2 & 956 \\
NM_001012632.1 & transcript variant 3 & 919 \\
NM_001012633.1 & transcript variant 4 & 897 \\
NM_001012634.1 & transcript variant 5 & 892 \\
NM_001012635.1 & transcript variant 6 & 880 \\
NM_001012636.1 & transcript variant 7 & 1093 \\
NM_001012718.1 & transcript variant 8 & 1243 \\
NM_001308078.1 & transcript variant 9 & \\
\hline
\end{tabular}

Table 2. Summary of the comparisons between the Intron: Exons of the nine transcript variants. These data also show which variants are more similar and designate which have deletions compared to transcript variant 1 . The corresponding protein sizes of each variant are also listed. Greek symbol designations were obtained from NCBI; designations were listed with the nucleotide sequence information

\begin{tabular}{lllll}
\hline Variant & $\begin{array}{l}\text { Nucleotides } \backslash \\
\text { protein sequence }\end{array}$ & Deletions/differences compared to Variant 1 & NCBI designation & Protein sizes \\
\hline $1,2,3,8$ & Similar to each & $\begin{array}{l}\text { variants } 2,8=\text { missing exon } 2 \\
\text { variant } 3=\text { missing exon } 1\end{array}$ & Isoform B $(\beta)$ & 188 \\
5,6 & Similar to each & variants $5,6=$ missing exon 1 and 5 & isoform C $(\varepsilon)$ & 168 \\
4 & Unique & variant $4=$ missing exon2 and part of exon 8 & Isoform A $(\alpha)$ & 131 \\
7 & Unique & variant $7=$ missing exons $1,2,6$ & Isoform D $(\delta)$ & 179 \\
$9(\mathrm{X})$ & Unique & variant $9=$ missing exon1; exons 4,5 combined; & Isoform E $(\gamma)$ & 234 \\
& & align of exon 8 show additional 145 nucleotides & & \\
\hline
\end{tabular}

Table 3. Densitometer analysis of PCR results comparing primer sets in MDA MB231 compared to MCF7. The variant lists those recognized by the particular primer set. The greatest level of differential expression are observed in Primer set $1 *$ and primer set $5^{* *}$

\begin{tabular}{llllll}
\hline Primer Sets(variants) & MDA mb231 (A) & PSn/Cont=A1 & MCF7 (B) & PSn/Cont = B1 & $($ A_n-B_n) \\
\hline PS1 $(1,2,3,5,6,8,9)$ & 3250.31 & 1.1876 & 1667.91 & 0.6134 & $0.5742^{*}$ \\
PS2 $(1,2,3,4,5,6,7,8,9)$ & 3566.82 & 1.3033 & 2706.17 & 0.9952 & 0.3081 \\
PS3 $(1,2,3,5,6,7,8,9)$ & 3872.77 & 1.4151 & 3291.91 & 1.2106 & 0.2045 \\
PS4 (1, 2, 4, 8) & 3155.86 & 1.1531 & 2497.12 & 0.9183 & 0.2348 \\
PS5 (9 Only) & 3127.19 & 1.1426 & 1710.03 & 0.6289 & $0.5137 * *$ \\
MRPL19 & 2736.79 & 2719.2 & & & \\
\hline
\end{tabular}

Supplemental Table 1. PCR primer sets. Table includes the primer sets, the variants detected using the particular primers, their corresponding sequences and amplicon sizes generated following PCR analyses

\begin{tabular}{llll}
\hline Primer Set (PS; variants) & Forward primer & Reverse primer & Amplicon length \\
\hline PS1 $(1,2,3,5,6,8,9)$ & AGCTGGAGGACGACTTCAAA & CCTGGAACCATCTCATGACC & $207 \mathrm{bp}$ \\
PS2 (All Variants) & CTTTCCAGTCCTACGGAGC & ACAGCACCAGGTCAGAGCT & $222 \mathrm{bp}$ \\
PS3 (All except 4) & CTGGGTGAAGGAGAAGGTG & GCAAAGGTGGTGTCAGTATC & $205 \mathrm{bp}$ \\
PS4 (Variants 1,2,4,8) & CCAGCTGTCCCGAGTCTGGA & AGAGAGACAGAGGTCTGGGGT & $174 \mathrm{bp}$ \\
PS5 9 (X) only & CCCTACTTCTGCTCAGGGGTT & CACCCACCTGTCCACGTCCT & $194 \mathrm{bp}$ \\
MRPL19 control & AAGCGTCGAAAAGGTCTTGA & GCTCAGGTTCCATGCTCATT & $306 \mathrm{bp}$ \\
\hline
\end{tabular}

Variants 1, 2, 3 and 8 correspond to IL32 $\beta$. Variants 5 and 6 correspond to IL32 $\varepsilon$. Variant 9 corresponds to IL $32 \gamma$. These data suggest that IL32 $\beta /$ IL $32 \varepsilon$ and IL32 $\gamma$ appear to contribute significantly to differential gene expression in our breast cancers. The least level of differential expression was observed using primer set three and primer set four (Table 3); these primer sets contained variants 4 or 7 . When primer sets contain either variants $4(\dot{\alpha})$ or $7(\delta)$, we observed the lowest levels of differential gene expression. Even though the transcripts were present, they appeared to contribute less to the processes affecting differential expression. Transcript variant 7 has fewer exons (i.e., more deletions) compared to all other transcript variants and variant 4 also contains substantial deletions. Transcript variant 4 also codes for the smallest of the proteins at 131 amino acids (Table 1 above). Based on their differential expression in the breast cell lines, the primer 
sets are ranked as primer set $1>$ primer set $5>$ primer set $2>$ primer set $4>$ primer set 3 . Overall, the PCR analysis and densitometer readings validate what we observed in the cell lines and patient samples (Player et al., 2014), in that IL32 is differentially expressed in the cell lines and even though their levels vary, several transcript variants contribute to the differential pattern of expression observed in these samples.

\section{Discussion}

To the best of our knowledge, this is the first attempt to characterize IL32 transcript variants in breast cancer cell lines. Comparatively little is known about the IL32 gene as it relates to expression in breast samples. Although the gene was identified over two decades ago in lymphocytes as a Natural Killer (NK4) protein, a search of PubMed using the terms 'breast and IL32' with or without the added term 'cancer' result in seven articles. This could partly be due to the perception that IL32 is involved in inflammatory events with little utility in breast cancers. Data supporting the role of other cytokines in breast cancers however have been documented. Studies show IL6/IL8 and TNF genes have clinical utility and together or individually the genes correlate with growth, survival and clinical prognoses (Hartman et al., 2013; Salgado et al., 2003; Tripsianis et al., 2014). Data generated from these studies has led to a shift in the paradigm related to the role of proinflammatory signaling processes in tumor progression (Atsumi et al., 2014). If data are consistent, IL32 is also involved in the pathogenesis of breast cancers validating the efforts to characterize the different transcript variants. Results from these data can then be useful for identifying variants to target for functional studies involved in understanding the role of IL32 in breast signaling pathways whether related to cancer or not.

The IL32 sequencing data has constantly evolved over the past few years emphasizing the need for current studies. Prior to year 2015 transcript variant 9 was designated as "X1"; it was updated in NCBI May 2015 at which time its name was changed to variant 9 and its sequence information was updated. An even more recent update (June 2016) of the transcript variant 9 sequence information resulted in an additional 267 nucleotides to the 3-prime region of the nucleotide sequence compared to X1. Although earlier studies likely did not include all of this information, investigators have compared the different IL32 transcript variants. Goda et al. (2006) found that IL $32 \beta$ was the most biologically active variant in activated T-cells and Choi et al. (2009) showed that IL32 $\gamma$ was the most biologically active in WISH epithelial cells following treatment with IFN- $\gamma$. Both IL32 $\beta$ and IL32 $\gamma$ are longer variants. The studies concluded that certain variants showed more biological activity, yet all variants show some degree of activity. At the time of these studies, six transcript variants had been identified. Measuring differential expression in breast cancer cell lines, we find the greatest levels of differentially expression when both IL $32 \beta$ (corresponding to variants 1, 2, 3 and 8) and IL32 $\gamma$ (which is probably variant 9 based on its size) are assessed. Variants 5 and 6 (ع) were also included with the IL32 $\beta / \gamma$ primer sets. These variants are shorter size transcript variants. Choi speculates that there is lower activity related to IL32 $\varepsilon$. We could not validate the performance of transcript variants 5 and 6 because we could not identify sequences unique to these variants that could be used for PCR analyses. Because all of the variants demonstrate some level of activity, we conclude that differential expression of IL32 in TNBC is not caused by a single transcript variant but a combination of variants. Consistent with previous studies, we also found that the other transcript variants showed some level of expression in breast cell lines.

In addition to examining variants with the most biological activity these same studies examined variants with the least level of biological activity (Goda et al., 2006; Choi et al., 2009). The investigators found that transcript variants with deletions (compared to transcript variant 1) code for proteins that display lower biological activity. Their data showed that IL32 $\alpha$ (referred to as 4 in this study) and IL32 $\delta$ (which likely corresponds to variant 7 in this study) demonstrated the least expression in WISH epithelial cells stimulate by IFN $\gamma$. Our data suggest that the shorter transcript variants 4 and 7 , both which contain substantial deletions, contribute less to differential gene expression compared to the longer transcript variants; the longest transcript variants being 1 and 9. Results of our study are consistent with previous studies in that we find that when relating biological activity to differential gene expression in breast cancer cells, shorter variants appear to contribute less to these processes. Our results did not address the contribution of proteins to these processes.

Both our and previous studies suggest that whether we are addressing transcript variants or biological activity, there seems to be a pattern in that the longer variants are more involved in the processes that drive these events. One possibility is that, overall, regardless of the tissue, variants with deletions lead to less functional molecules. Or it could be that the shorter variants have particular biological events that they 'drive' which are yet to be described. This is consistent with the popular theory that transcript variants have a purpose and certainly a function if the particular protein product is generated (Keren et al., 2010; Sohail and Xie, 2015; Wang et al., 2008).

\section{Conclusion}

We have characterized the nine IL32 transcript variants in breast cell lines and show that the nucleotide sequences code for five different proteins. There is 
incredible sequence similarity between the different variants and variants with longer nucleotide sequences appear to contribute more to the differential gene expression observed between our samples. We also conclude that many of the other transcript variants show some contribution to gene expression in a TNBC cell line and because all of the IL32 transcript variants demonstrate some level of activity, it will be difficult to single-out one variant to possibly target as an attempt to modulate the gene's activity. Based on sequences retrieved from NCBI, our data suggest that in the event IL32 gene is targeted for study of differential gene expression in the breast cancer cell lines, investigators should consider strategies that target a combination of transcript variants.

\section{Acknowledgment}

The authors would like to thank the Department of Biology and the Office of Research for their partial funding in support of this project. The authors would also like to acknowledge the continuous support of the microarray facility of the microarray facility $(m A d b)$ of the National Cancer Institute (Bethesda MD)

\section{Author's Contributions}

The project was conceived by AP. KB performed the experiments and AP and KB performed the data analyses and wrote the manuscript.

\section{Disclosure} work.

The authors report no conflicts of interest in this

Supplemental Data-Table 1: IL32 primer sequences used for analyses of the IL32 1 to 9 transcript variants.

\section{References}

Atsumi, T., R. Singh, L. Sabharwal, H Bando and J. Meng et al., 2014. Inflammation amplifier, a new paradigm in cancer biology. Cancer Res., 74: 8-14. DOI: 10.1158/0008-5472.CAN-13-2322

Choi, J.D., S.Y. Bae, J.W. Hong, T. Azam and C.A. Dinarello et al., 2009. Identification of the most active interleukin-32 isoform. Immunology, 126: 535-542. DOI: 10.1111/j.1365-2567.2008.02917.x

Corpet, F., 1988. Multiple sequence alignment with hierarchical clustering. Nucleic Acids Res., 16: 10881-10890. DOI: 10.1093/nar/16.22.10881

Dahl, C.A., R.P. Schall, H.L. He and J.S. Cairns, 1992. Identification of a novel gene expressed in activated natural killer cells and T cells. J. Immunol., 148: 597-603. PMID: 1729377
Felaco, P., M.L. Castellani, M.A. De Lutiis, M. Felaco and F. Pandolfi et al., 2009. IL-32: A newly-discovered proinflammatory cytokine. J. Biol. Regul. Homeost, 23: 141-147. PMID: 19828090

Goda, C., T. Kanaji, S. Kanaji, G. Tanaka and K. Arima et al., 2006. Involvement of IL-32 in activation-induced cell death in T cells. Int. Immunol., 18: 233-240. DOI: 10.1093/intimm/dxh339

Hartman, Z.C., G.M. Poage, P. den Hollander, A. Tsimelzon and J. Hill et al., 2013. Growth of triplenegative breast cancer cells relies upon coordinate autocrine expression of the proinflammatory cytokines IL-6 and IL-8. Cancer Res., 73: 3470-3480. DOI: 10.1158/0008-5472.CAN-12-4524-T

Ishigami, S., T. Arigami, Y. Uchikado, T. Setoyama and Y. Kita et al., 2013. IL-32 expression is an independent prognostic marker for gastric cancer. Med. Oncol., 30: 472-472. DOI: 10.1007/s12032-013-0472-4

Joosten, L.A., B. Heinhuis, M.G. Netea and C.A. Dinarello, 2013. Novel insights into the biology of interleukin-32. Cell Mol. Life Sci., 70: 3883-3892. DOI: 10.1007/s00018-013-1301-9

Kang, J.W., S.C. Choi, M.C. Cho, H.J. Kim and J.H. Kim et al., 2009. A proinflammatory cytokine interleukin-32 $\beta$ promotes the production of an anti-inflammatory cytokine interleukin-10. Immunology, 128: e532-540. DOI: $10.1111 / j .1365-2567.2008 .03025 . x$

Keren, H., G. Lev-Maor and G. Ast, 2010. Alternative splicing and evolution: Diversification, exon definition and function. Nat. Rev. Genet, 11:345-355. DOI: $10.1038 / \mathrm{nrg} 2776$

Kim, S.H., S.Y. Han, T. Azam, D.Y. Yoon and C.A. Dinarello, 2005. Interleukin-32: A Cytokine and Inducer of TNF $\alpha$. Immunity, 22: 131-142. DOI: 10.1016/j.immuni.2004.12.003

Kim, S.H., T. Azam, D. Novick, D.Y. Yoon and L.L. Reznikov et al., 2002. Identification of amino acid residues critical for biological activity in human interleukin-18. J. Biol. Chem., 277: 10998-11003. DOI: $10.1074 /$ jbc.M108311200

Kobayashi, H., E.M. Yazlovitskaya and P.C. Lin, 2009. Interleukin-32 positively regulates radiationinduced vascular inflammation. Int. J. Radiat. Oncol. Biol. Phys., 74: 1573-1579. DOI: 10.1016/j.ijrobp.2009.04.017

Mabilleau, G. and A. Sabokbar, 2009. Interleukin-32 promotes osteoclast differentiation but not osteoclast activation. PLoS One, 4: e4173-e4173. DOI: 10.1371 /journal.pone.0004173

Nold-Petry, C.A., I. Rudloff, Y. Baumer, M. Ruvo and D. Marasco et al., 2014. IL-32 promotes angiogenesis. J. Immunol., 192: 589-602. DOI: $10.4049 /$ jimmunol.1202802 
Oh, J.H., J.O. Yang, Y. Hahn, M.R. Kim and S.S. Byun et al., 2005. Transcriptome analysis of human gastric cancer. Mamm. Genome, 16: 942-954. DOI: $10.1007 / \mathrm{s} 00335-005-0075-2$

Park, J.S., S.Y. Choi, J.H. Lee, M. Lee and E.S. Nam et al., 2013. Interleukin-32 $\beta$ stimulates migration of MDAMB-231 and MCF-7cells via the VEGF-STAT3 signaling pathway. Cell Oncol., 36: 493-503. DOI: $10.1007 / \mathrm{s} 13402-013-0154-4$

Player, A., T. Oguamanam, J. Okanmelu, K. Burrell and M. Hollomon, 2014. Preliminary characterization of IL32 in basal-like/triple negative compared to other types of breast cell lines and tissues. BMC Res. Notes, 7: 501-501. DOI: 10.1186/1756-0500-7-501

Rozen, S. and H. Skaletsky, 2000. Primer3 on the WWW for general users and for biologist programmers. Methods Mol. Biol., 132: 365-386. PMID: 10547847

Salgado, R., S. Junius, I. Benoy, P. Van Dam and P. Vermeulen et al., 2003. Circulating interleukin-6 predicts survival in patients with metastatic breast cancer. Int. J. Cancer, 103: 642-646. DOI: $10.1002 / \mathrm{ijc} .10833$

Sohail, M. and J. Xie, 2015. Evolutionary emergence of a novel splice variant with an opposite effect on the cell cycle. Mol. Cell Biol., 35: 2203-2214.

DOI: $10.1128 / \mathrm{MCB} .00190-15$
Tripsianis, G., E. Papadopoulou, K. Anagnostopoulos, S. Botaitis and M. Katotomichelakis et al., 2014. Coexpression of IL- 6 and TNF- $\alpha$ : Prognostic significance on breast cancer outcome. Neoplasma, 61: 205-212. PMID: 24299316

Trojan, L., A. Schaaf, A. Steidler, M. Haak and G. Thalmann et al., 2005. Identification of metastasisassociated genes in prostate cancer by genetic profiling of human prostate cancer cell lines. Anticancer Res., 25: 183-191. PMID: 15816537

Wang, E.T., R. Sandberg, S. Luo, I. Khrebtukova and L. Zhang et al., 2008. Alternative isoform regulation in human tissue transcriptomes. Nature, 456: 470-476. PMID: 18978772

Wang, S., F. Chen and L. Tang, 2015. IL-32 promotes breast cancer cell growth and invasiveness. Oncol. Lett., 9: 305-307.

Yue, D., Y. Wang, P. Ma, Y.Y. Li and H. Chen et al., 2010. Effects of transferred NK4 gene on proliferation, migration, invasion and apoptosis of human prostate cancer DU145 cells. Asian J. Androl., 12: 381-389. DOI: 10.1038/aja.2010.22 